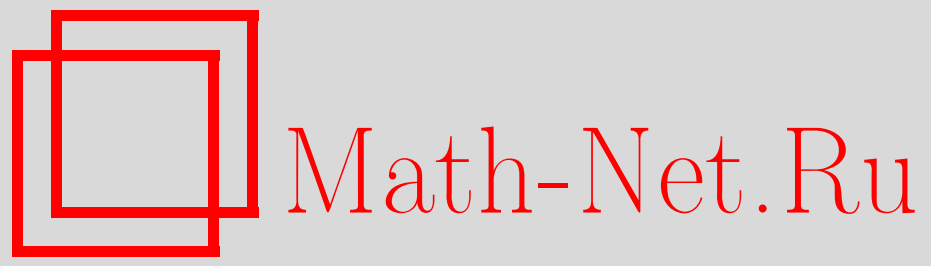

С. В. Савченко, Гомологические неравенства для конечных топологических цепей Маркова, Функи. анализ и его прил., 1999, том 33, выпуск 3, 91-93

DOI: https://doi.org/10.4213/faa375

Использование Общероссийского математического портала MathNet.Ru подразумевает, что вы прочитали и согласны с пользовательским соглашением

http://www.mathnet.ru/rus/agreement

Параметры загрузки:

IP: 3.85 .73 .92

26 апреля 2023 г., 09:17:15

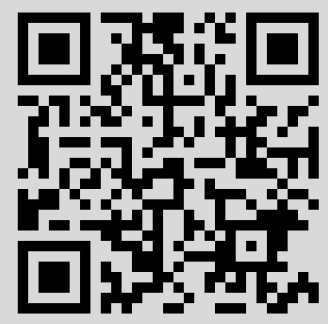


УДК $517.987 .5+517.938$

\title{
Гомологические неравенства для конечных топологических цепей Маркова*
}

\author{
(C) 1999. C. В. САвченко
}

Пусть $X^{+}$- множество последовательностей $x=\left\{x_{i}\right\}_{i=0}^{\infty}$, соответствующих односторонне-бесконечным путям конечного связного апериодического графа с $D$ вершинами и матрицей смежности $A$, и $S$ - отображение сдвига влево: $S x=x^{\prime}$, где $x_{i}^{\prime}=x_{i+1}$. В тихоновской топологии прямого произведения множество $X^{+}$является компактом, а $S$ - непрерывным конечнократным ( $\left.\operatorname{card} S^{-1} x \leqslant D\right)$ сюръективным отображением. Пара $\left(S, X^{+}\right)$называется односторонней топологической цепью Маркова. Обозначим через $C\left(X^{+}\right)$пространство непрерывных функций на $X^{+}$и для любого $\psi \in C\left(X^{+}\right)$определим оператор Рюэля $L_{\psi}$ на функциях $g \in C\left(X^{+}\right)$по следующему правилу:

$$
\left(L_{\psi} g\right)(x)=\sum_{x^{\prime} \in S^{-1} x} e^{\psi\left(x^{\prime}\right)} g\left(x^{\prime}\right) .
$$

Лемма 1. Пусть для $f \in C\left(X^{+}\right)$существует положительная функиия $h$, удовлетворяюшая уравнению $L_{-f} h=\lambda h$ с некоторым $\lambda>0$. Тогда функиия $f(x)-\ln h(x)+\ln h(S x)+\ln \lambda$ неотричательна.

ДокАЗАТЕЛЬСтво. Нетрудно проверить, что если $L_{-f} h=\lambda h$ и $h>0$, то $L_{-f^{\prime}} \mathbf{1}=\mathbf{1}$, где $f^{\prime}(x)=f(x)-\ln h(x)+\ln h(S x)+\ln \lambda$ и $\mathbf{1}$ - функция на $X^{+}$, тождественно равная единице. По определению оператора Рюэля (см. (1)) последнее равенство возможно только тогда, когда функция $f^{\prime}$ неотрицательна. Лемма доказана.

Обозначим через $I\left(X^{+}\right)$множество инвариантных относительно $S$ вероятностных мер на пространстве $X^{+}$и положим $P(f)=\sup \left\{h_{\mu}(S)+\int f d \mu: \mu \in\right.$ $\left.I\left(X^{+}\right)\right\}$, где $h_{\mu}(S)$ - энтропия отображения $S$ относительно меры $\mu$.

Пусть $B_{m}(x)=\left\{x^{\prime} \in X^{+}: x_{0}^{\prime}=x_{0}, \ldots, x_{m}^{\prime}=x_{m}\right\}$. Для $f \in C\left(X^{+}\right)$положим $|f|_{\infty}=\max \left\{|f(x)|: x \in X^{+}\right\}, \operatorname{var}_{m} f=\max \left\{\left|f(x)-f\left(x^{\prime}\right)\right|: x \in X^{+}, x^{\prime} \in B_{m}(x)\right\}$ и $|f|_{\theta}=\sup \left\{\theta^{-m} \operatorname{var}_{m} f: m \geqslant 0\right\}$, где $\theta \in(0,1)$. Если $|f|_{\theta}<\infty$, то функция $f$ является гёльдеровской относительно метрики $d_{\theta}\left(x, x^{\prime}\right)=\theta^{N\left(x, x^{\prime}\right)}$, где $N\left(x, x^{\prime}\right)=\inf \left\{m: x_{m} \neq x_{m}^{\prime}\right\}$.

Две непрерывные функции $f(x)$ и $g(x)$, для которых существует такая непрерывная функция $u(x)$, что $f(x)=g(x)-u(x)+u(S x)$, называются гомологичными. В [1] и [2] было показано, что если сумма сдвигов гёльдеровской функции $f$ вдоль любой периодической траектории равна нулю (это условие формально слабее, но, на самом деле, эквивалентно требованию равенства нулю интеграла $\int f d \mu$ для любого $\mu \in I\left(X^{+}\right)$), то она гомологична нулю (этот результат известен теперь как теорема Лившица). В нашей работе мы фактически рассмотрим вопрос о гомологичности неотрицательным и положительным функциям. Положим $c(f)=\min \left\{\int f d \mu: \mu \in I\left(X^{+}\right)\right\}$. 
ТеОРема 1. Пусть $f$ - гёльдеровская функиия на пространстве $X^{+}$c $|f|_{\theta}<\infty$. Тогда существует гёльдеровская функиия и $c|u|_{\theta}<\infty$, такая, что

$$
f(x)-u(x)+u(S x) \geqslant c(f) .
$$

ДокАЗАТЕльство. Выберем произвольную последовательность положительных чисел $\alpha_{n}$, стремящихся к бесконечности. Так как для любой гёльдеровской функции $\psi$ существует положительное гёльдеровское решение $h$ уравнения $L_{\psi} h=e^{P(\psi)} h$ (см. [3, теорема 1.7]; равенство $P(\psi)=\ln \lambda$ следует из доказательства теоремы 1.16 в [3]), то при любом $n$ можно определить функцию $f_{n}^{\prime}(x) \equiv f(x)+\alpha_{n}^{-1} P\left(-\alpha_{n} f\right)-\alpha_{n}^{-1} \ln h_{n}(x)+\alpha_{n}^{-1} \ln h_{n}(S x)$, где $h_{n}$ - положительное решение уравнения

$$
L_{-\alpha_{n} f} h_{n}=e^{P\left(-\alpha_{n} f\right)} h_{n} .
$$

В силу леммы 1 она неотрицательна. В [3] при доказательстве леммы 1.8 было показано, что для любой гёльдеровской функции $\psi$ с $|\psi|_{\theta}<\infty$ решение $h$ уравнения $L_{\psi} h=e^{P(\psi)} h$ удовлетворяет следующему неравенству:

$$
h(x) \leqslant h\left(x^{\prime}\right) \exp \left(2|\psi|_{\theta}(1-\theta)^{-1} \theta^{m+1}\right) \quad \text { при } x^{\prime} \in B_{m}(x) .
$$

Кроме того, умножая, если надо, $h$ на подходящую константу, можно добиться справедливости неравенства

$$
K^{-1} \leqslant h(x) \leqslant K,
$$

где $\ln K=M P(\psi)+M|\psi|_{\infty}+2|\psi|_{\theta}(1-\theta)^{-1} \theta$ и $M$ - любое натуральное число, при котором все элементы матрицы $A^{M}$ положительны. В нашем случае $\psi=$ $-\alpha_{n} f$. Поэтому

$$
\begin{gathered}
h_{n}(x) \leqslant h_{n}\left(x^{\prime}\right) \exp \left(2 \alpha_{n}|f|_{\theta}(1-\theta)^{-1} \theta^{m+1}\right) \text { при } x^{\prime} \in B_{m}(x), \\
K_{n}^{-1} \leqslant h_{n}(x) \leqslant K_{n},
\end{gathered}
$$

где $\ln K_{n}=M P\left(-\alpha_{n} f\right)+\alpha_{n} M|f|_{\infty}+2 \alpha_{n}|f|_{\theta}(1-\theta)^{-1} \theta$.

В силу $\left(2^{\prime}\right)$ имеем

$$
\left|\alpha_{n}^{-1} \ln h_{n}(x)-\alpha_{n}^{-1} \ln h_{n}\left(x^{\prime}\right)\right| \leqslant 2|f|_{\theta}(1-\theta)^{-1} \theta^{m+1} \quad \text { при } x^{\prime} \in B_{m}(x) .
$$

Таким образом, $\left|\alpha_{n}^{-1} \ln h_{n}\right|_{\theta} \leqslant 2|f|_{\theta}(1-\theta)^{-1} \theta$. В силу $\left(3^{\prime}\right)$ справедливо неравенство $-\alpha_{n}^{-1} \ln K_{n} \leqslant \alpha_{n}^{-1} \ln h_{n}(x) \leqslant \alpha_{n}^{-1} \ln K_{n}$, и, следовательно, $\left|\alpha_{n}^{-1} \ln h_{n}\right|_{\infty} \leqslant$ $\alpha_{n}^{-1} M P\left(-\alpha_{n} f\right)+M|f|_{\infty}+2|f|_{\theta}(1-\theta)^{-1} \theta$. Без ограничения общности можно считать, что $c(f)=0$. В этом случае $0 \leqslant P\left(-\alpha_{n} f\right) \leqslant P(0) \leqslant \ln D$. Поэтому последовательность функций $\alpha_{n}^{-1} \ln h_{n}$ ограничена по норме $\|\cdot\|_{\theta} \equiv$ $|\cdot|_{\infty}+|\cdot|_{\theta}$. По теореме Асколи-Арцела существует такая последовательность номеров $n_{k}$, что последовательность функций $\alpha_{n_{k}}^{-1} \ln h_{n_{k}}$ сходится по норме $|\cdot|_{\infty}$ к гёльдеровской функции $u$ с $\|u\|_{\theta} \leqslant M|f|_{\infty}+4|f|_{\theta}(1-\theta)^{-1} \theta$. Соответственно последовательность неотрицательных функций $f_{n_{k}}^{\prime}(x)$ сходится к неотрицательной гёльдеровской функции $f(x)-u(x)+u(S x)$. В заключение заметим, что если функция $f$ зависит только от $n+1$ координат $x_{0}, \ldots, x_{n}$, то функция $u(x)$ будет зависеть от $n$ координат (см. [4]). Теорема доказана.

ЗАмечание 1 . Теорема 1 была сформулирована В. Пэрри в качестве гипотезы и доказана Пуном [5] для функций, зависящих от конечного числа координат и принимающих только целочисленные значения. 
СЛЕДСТВИЕ 1. Пусть $f \in C\left(X^{+}\right)$. Тогда для любого $\varepsilon>0$ существует такая функиия $u_{\varepsilon}$, зависящая от конечного числа $n=n(f, \varepsilon)$ координат, что

$$
f(x)-u_{\varepsilon}(x)+u_{\varepsilon}(S x)>c(f)-\varepsilon .
$$

ДокаЗАТЕЛЬСтво. Положим $f_{n}(x)=\min \left\{f\left(x^{\prime}\right): x^{\prime} \in B_{n}(x)\right\}$. Очевидно, функция $f_{n}(x)$ зависит только от первых $n+1$ координат. По теореме Кантора о равномерной непрерывности функции, непрерывной на метрическом компакте, $n$ можно выбрать так, чтобы $\left|f-f_{n}\right|_{\infty}<\varepsilon$. Теорема 1 гарантирует существование такой функции $u_{\varepsilon}$, зависящей от $n$ координат, что $f_{n}(x)-u_{\varepsilon}(x)+$ $u_{\varepsilon}(S x) \geqslant c\left(f_{n}\right)$. В силу выбора $f_{n}$ функция $f-f_{n}$ неотрицательна. Поэтому минимум функции $f(x)-u_{\varepsilon}(x)+u_{\varepsilon}(S x)=\left(f(x)-f_{n}(x)\right)+\left(f_{n}(x)-u_{\varepsilon}(x)+u_{\varepsilon}(S x)\right)$ не меньше, чем $c\left(f_{n}\right)>c(f)-\varepsilon$. Следствие доказано.

ЗАмечание 2. Результаты теоремы 1 и следствия 1 без труда переносятся на случай пространства $X$ двусторонне-бесконечных последовательностей. При этом для доказательства «двустороннего» аналога теоремы 1 нужно воспользоваться тем фактом, что любая гёльдеровская функция на пространстве $X$ гомологична гёльдеровской функции, зависящей только от координат с неотрицательными номерами (см. [6]).

Автор благодарит участников семинара МГУ по динамическим системам под руководством акад. Д. В. Аносова и проф. А. М. Степина, которыми в ноябре 1995 г. была сформулирована задача о гомологичности положительным функциям. Автор также признателен проф. М. Полликоту и д-ру Р. Шарпу за предоставление ссылок и необходимых сведений по истории вопроса.

\title{
ЛИТЕРАТУРА
}

1. Лившии А. Н. Матем. заметки, 10, № 5, 555-564 (1971). 2. Лившии А. Н. Изв. АН СССР, Сер. матем., 36, № 6, 1296-1320 (1972). 3. Боуэн Р. Методы символической динамики. Сб. Математика. Мир, М., 1979. 4. Parry W., Pollicott M. Asterisque, 187-188, 1-268 (1990). 5. Poon Y-T. Trans. Amer. Math. Soc., 311, 515-533 (1989). 6. Синай Я. Г.УМН, 27, № 4, 21-64 (1972).

Институт теоретической физики им. Л. Д. Ландау РАН e-mail: sav@icp.ac.ru

Поступило в редакцию 12 марта 1998 г.

\section{УДК 513.88}

\section{Гладкие изометричные погружения в бесконечномерную сферу*}

\author{
(c) 1999. И. Г. ЦАРьков, Е. Т. ШАвГУлидЗЕ
}

Работа посвящена равномерно гладким погружениям и вложениям бесконечномерных банаховых пространств в произвольные окрестности нуля, причем в случае гильбертова пространства удается построить равномерно гладкое изометричное погружение в гильбертову сферу. Эта задача восходит к известным

*Работа выполнена при финансовой поддержке Российского фонда фундаментальных исследований (гранты 96-01-00378, 97-01-00228). 